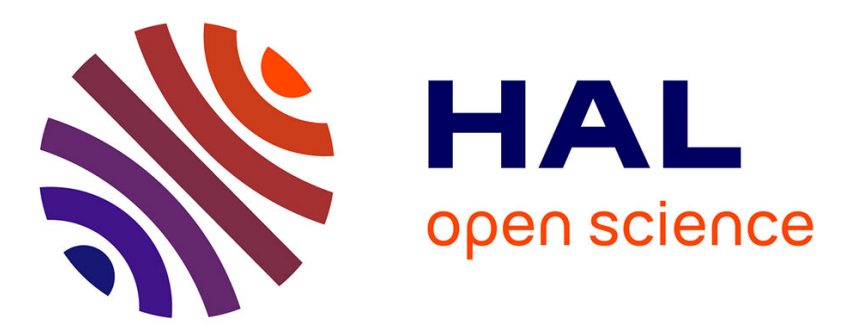

\title{
Maximal resultant four fingertip force and fatigue of the extrinsic muscles of the hand in different sport climbing finger grips
}

Franck Quaine, Laurent Vigouroux

\section{- To cite this version:}

Franck Quaine, Laurent Vigouroux. Maximal resultant four fingertip force and fatigue of the extrinsic muscles of the hand in different sport climbing finger grips. International Journal of Sports Medicine, 2004. hal-01635483

\section{HAL Id: hal-01635483 \\ https://hal.science/hal-01635483}

Submitted on 15 Nov 2017

HAL is a multi-disciplinary open access archive for the deposit and dissemination of scientific research documents, whether they are published or not. The documents may come from teaching and research institutions in France or abroad, or from public or private research centers.
L'archive ouverte pluridisciplinaire HAL, est destinée au dépôt et à la diffusion de documents scientifiques de niveau recherche, publiés ou non, émanant des établissements d'enseignement et de recherche français ou étrangers, des laboratoires publics ou privés. 


\section{Maximal Resultant Four Fingertip Force and Fatigue of the Extrinsic Muscles of the Hand in Different Sport Climbing Finger Grips}

\section{Abstract}

This study investigates the effect of simulated sport climbing finger grips on the resultant four fingertip force and the rate of $\mathrm{fa}$ tigue of finger flexor muscles. Six elite sport climbers sat on a chair with the right forearm placed in a handgrip dynamometer modified so that only the fingertips applied direct force. They were asked to perform three maximal voluntary contractions (MVC). After ten minutes, they had to reach $80 \%$ of the peak MVC intermittently with a $5 \mathrm{~s}$ contraction followed by $5 \mathrm{~s}$ of rest for 20 repetitions. Two common sport climbing finger grips were tested: the "slope" grip and the "crimp" grip. In the "crimp" grip, the distal interphalangeal joint (DIP) is hyper-extended and the proximal interphalangeal joint (PIP) is flexed from $90^{\circ}$ to $100^{\circ}$ In the "slope" grip, DIP is flexed from $50^{\circ}$ to $70^{\circ}$ and PIP is flexed just slightly. The surface EMG of the hand extrinsic flexors and the maximal resultant four fingertip force were recorded. Results show that the maximal resultant four-fingertip force does not depend on the type of finger grips (on average $420 \mathrm{~N}, \mathrm{p}>0.05$ ). EMG median frequency of finger flexor muscles and resultant four fingertip force rate decrease are similar between both sport climbing finger grips ( $p>0.05$ ). This shows that the fatigue rate is not dependent upon the sport climbing finger grips. In conclusion, the results suggest that the use of the "crimp" or the "slope" grip does not provide any benefit with respect to muscular fatigue in sport climbing.

\section{Key words}

Sport climbing · finger · biomechanics · electromyography

\section{Introduction}

Sport climbing was traditionally one aspect of mountaineering [8]. This activity presents a remarkable development in scope and quality as a recreational activity and competitive event [6] Indoor sport climbing walls are set up to accommodate varying route skill levels by modifying wall angles and hold sizes. In order to achieve the routes climbers have to be able to maintain extreme force on their digital extremities despite the small size of the holds and the muscular fatigue [16].

Sport climbing finger grips result from a variety of finger joint positions. The most common are the "crimp" grip and the "slope" grip. In the "crimp" grip, the distal interphalangeal joint (DIP) is hyper-extended and the proximal interphalangeal joint (PIP) is flexed from $90^{\circ}$ to $100^{\circ}$. In the "slope" grip, DIP is flexed from $50^{\circ}$ to $70^{\circ}$ and PIP is flexed just slightly [16]. Schweizer [16] showed that the maximum force at the middle fingertip is not significantly different for the "slope" grip in comparison with the "crimp" grip ( $82 \mathrm{~N}$ versus $78 \mathrm{~N}$ ). However, this author did not monitor the forces applied by all the fingers concomitantly involved in the grip. The question remains unclear whether the resultant fingertip force (i.e. the sum of the forces applied by the four fingertips) varies according to the climbing finger grips.

Few studies have reported muscular fatigue data concerning finger flexors in sport climbers. Fergusson and Brown [5] measured endurance in both sustained and rhythmic isometric handgrip

\section{Affiliation}

EA 597, UFR APS, Université Joseph Fourier, Grenoble, France

Correspondence

Franck Quaine -UFR APS, Université Joseph Fourier - 1741 rue de la Piscine - BP 53x, 38041, Grenoble

Cedex 9 · France · Phone: + 330476514593 · Fax: + 330476514469 · E-mail: franck.quaine@ujf-grenoble.fr

Accepted after revision: October 20, 2003

Bibliography

Int J Sports Med 2004; 25: 634-637 10 Georg Thieme Verlag KG . Stuttgart · New York.

DOI 101055/s-2004-821117. Published online July 26, 2004

ISSN $0172-4622$ 
exercises at $40 \%$ of maximum grip force. They reported no significant different endurance times between climbers and nonclimbers during sustained contraction, while the intermittent time for climbers was twice as long as for non-climbers. Surface electromyography (EMG) is currently used to analyse fatigue during static intermittent sub-maximal voluntary muscle contractions $[2,7,17]$. The frequency components of the surface EMG are a function of the duration of motor unit action potentials, the geometry of the surface electrodes, the degree of motor unit synchronisation and the conduction velocity of action potentials on the sarcolemma. They are relatively independent from the tension exerted by the muscle. During fatiguing contractions, there is an increase in the amplitude of the low-frequencies and a reduction in the amplitude of the high frequencies components of the EMG [11].

No fatigue experiments based on EMG analysis have been performed to assess muscular fatigue associated with different sport climbing finger grips. The aim of this experiment was to measure the effect of sport climbing finger grips on the maximal resultant four fingertip force and fatigue rates of the extrinsic muscles of the hand in a simulated sport climbing gripping task.

\section{Materials and Methods}

Previous work [13] showed that high postural constraints associated with balance in sport climbing induce significant intra and inter-subject supporting force variability. Consequently, subjects were seated and instructed to keep their forearm on a table with the hand in pronation (Fig.1). In that case, the postural constraints are drastically reduced in comparison to a sportclimbing posture [10] and it is easy to control and modify the finger grip similarly for each subjects.

The wrist was fixed by a mitten at $40^{\circ}$ of extension in front of the hold. The upper arm was at $45^{\circ}$ of abduction, the elbow joint being flexed at $90^{\circ}$. The hold consisted of a steel plate $(100 \times 20 \times 3 \mathrm{~mm})$ covered with a non-skid surface fastened to the force sensor. The fingers gripped the hold on a one centimetre deep surface. The thumb did not act as an additional gripping force. A mono-axial load cell (Slumberger, model CD-750, France) was used to measure the resultant grip force applied by the fingers. The signal was amplified (PM, model 1965, France) before recording.

The tests were conducted in the "crimp" and "slope" grips described by Schweizer [16]. In the present study, in the "crimp" grip, DIP is hyper-extended (mean: $-30^{\circ} \mathrm{SD}: 9^{\circ}$ ) and PIP is flexed (mean: $90^{\circ} \mathrm{SD}: 16^{\circ}$ ). In the "slope" grip, DIP is flexed (mean: $60^{\circ}$ SD: $12^{\circ}$ ) and PIP is flexed (mean: $20^{\circ}$ SD: $11^{\circ}$ ). The angles were controlled in the sagittal plane using a digital camera (Sony, DSC-S70, Japan). No data were recorded in the top view since the fingers were aligned with the longitudinal axis of the hand.

Surface EMG activity of the hand extrinsic flexors (i.e. flexor digitorum superficialis and profundus, FDS and FDP) and extensor (i.e. extensor digitorum communis, EDC) was recorded. Pre-amplifier $(\times 600) \mathrm{Ag}-\mathrm{AgCl}$ electrodes (Graphic Controls, Canada) were used (input impedance $10 \mathrm{G} \Omega$, common mode rejection ra-

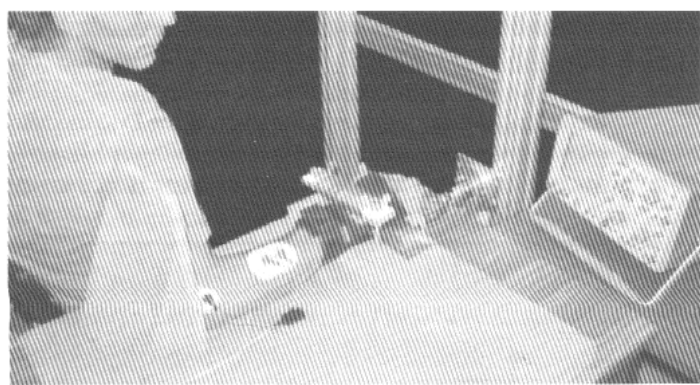

Fig. 1 Photo of the experimental setup. The subject controlled the resultant four fingertip force applied to the hold using the oscilloscope.

tio $=100 \mathrm{db}$ at $50 / 60 \mathrm{~Hz}$, bandwidth $=5-1000 \mathrm{~Hz}$ ). The surface electrodes were placed as suggested by Blackwell et al. [3]. This procedure allows the recording of EMG activity of both FDP and FDS. To ensure that the flexor carpi radialis (a wrist flexor) was not contributing significantly to the EMG signal, the position of the electrodes was considered appropriate when EMG signal was present during finger flexion with a voluntary stable wrist, yet absent during wrist flexion with no voluntary finger flexion. For the extensor muscles, the electrodes were placed around the $1 / 4$ point on a line drawn from the lateral epicondyle to the styloid process of the ulna [1]. EMG activity of the EDC was used to control EDC involvement during the exercise. As previously shown [14] the ratio of flexor and extensor EMG amplitude was constant throughout the exercise in each sport-climbing grip, which suggests a constant co-activation throughout the exercise. Moreover, Mogk and Keir [9] analysed EMG cross talk to evaluate the magnitude of common signal present between electrode pairs around the forearm. These authors concluded that less than $2 \%$ of common signal was present between flexor and extensors electrode pairs, attesting a low cross talk between these muscles. All force and EMG signals were sampled at $1024 \mathrm{~Hz}$. The raw EMG were low pass filtered $(300 \mathrm{~Hz}$ cutoff, zero lag fourth order Butterworth filter, Mazet Electronique, Biostim 6082, France).

Subjects warmed up on the device by carrying out a series of 100 contractions for $5 \mathrm{~s}$ at $10 \mathrm{~N}$ as advised by Schweizer [16]. The experimenter controlled that the warm-up exercise did not induce fatigue. After a 3 min rest, subjects performed three maximal isometric finger flexion contractions for $5 \mathrm{~s}$ separated by a $5 \mathrm{~min}$ resting period. The highest peak force was adopted as maximal voluntary contraction force (MVC). After ten minutes rest, subjects carried out twenty finger flexion contractions at $80 \%$ MVC during $5 \mathrm{~s}$ followed by $5 \mathrm{~s}$ of rest [12]. This protocol mimics the standard of actual difficult routes which requires an intermittent exercise at an intense level of muscle contraction $[5,12]$ during twenty contractions. The number of contractions corresponds to twenty climbing movements, which corresponds approximately to the standard difficult routes tested by Watts [18].

Each subject was provided with an ongoing force output displayed on a oscilloscope to confirm his achievement throughout the test. 
Table 1 Presentation of individual performances in the "crimp" grip and in the "slope" grip. "MVC" represents the maximal resultant four fingertip force. "20th contraction" represents the resultant four fingertip force recorded at the 20th contraction of the fatiguing protocol. " $f_{\text {med }}$ slopes" represents the slope of the EMG median frequency of the flexor muscle during the fatiguing protocol

\begin{tabular}{|c|c|c|c|c|c|c|}
\hline & “Crimp" MVC & "Slope" MVC & $\begin{array}{l}\text { “Crimp" } \\
\text { 20th contraction }\end{array}$ & $\begin{array}{l}\text { "Slope" } \\
\text { 20th contraction }\end{array}$ & "Crimp" $f_{\text {med }}$ slopes & "Slope" $f_{\text {med }}$ slopes \\
\hline Subject 1 & 412.86 & 366.6 & 224.22 & 195.89 & -0.96 & 0.1 \\
\hline Subject 2 & 440.21 & 429.63 & 296.41 & 270.1 & -0.58 & -0.32 \\
\hline Subject 3 & 386.7 & 449.16 & 292.48 & 345.71 & -0.58 & -1.28 \\
\hline Subject 4 & 385.5 & 434 & 295.59 & 236.38 & -0.76 & -1.7 \\
\hline Subject 5 & 383.51 & 487 & 307.14 & 353.4 & -0.29 & -0.87 \\
\hline Subject 6 & 437.2 & 439.46 & 207.13 & 274.02 & -1.41 & -0.87 \\
\hline Mean & 407.66 & 434.3 & 270.49 & 279.25 & -0.76 & -0.82 \\
\hline$(S D)$ & (26.35) & $(39.06)$ & (39.33) & (61.34) & $(0.38)$ & $(0.62)$ \\
\hline
\end{tabular}

Force and EMG were divided into two-second segments adjusted two seconds after the onset of the force increase. For each subject, median frequencies $\left(f_{\text {med }}\right)$ were computed using a Fast Fourier Transform (FFT) for each two-second segment. They were expressed relative to the $f_{\text {med }}$ value monitored during MVC tests. The time-related behaviour of $f_{\text {med }}$ was plotted and the slope of the line of best fit was computed for each subject. Slopes were averaged across all subjects and used as fatigue index. All computation procedures were performed using Matlab software (The Math Works, Inc., USA).

Descriptive statistics are means (SD) in the text and means (SEM) in the figure. Dependent group $t$-tests of significance were used to identify differences between the "crimp" and "slope" grip in maximal grip forces, in 20th force intensities and in the slopes of $f_{\text {med. }}$. An alpha level of 0.05 was used for all statistical tests.

\section{Results}

Individual performance of the six subjects is presented in Table $\mathbf{1}$. Two subjects (subjects 1 and 2) present greater maximal resultant four fingertip force during the "crimp" grip whereas three subjects (subjects $3,4,5$ ) present a greater performance in the "slope" grip and one subject presents similar performance in both grips (subject 6 ). The comparison of the average maximal resultant four fingertip forces indicates that the values did not present significant differences between the "crimp" and the "slope" grips $(p>0.05)$. The maximum force amounted to $407.66 \mathrm{~N}$ (SD: $26.35 \mathrm{~N}$ ) in the "crimp" grip, whereas it equalled $434.3 \mathrm{~N}$ (SD: $39.06 \mathrm{~N}$ ) in the "slope" grip.

The fatiguing protocol results in a decrease of resultant four fingertip force for each subject whatever the climbing finger grip. A more pronounced decrease was observed for subject 6 in the "crimp" grip (from $437.2 \mathrm{~N}$ to $207.13 \mathrm{~N}$ ) and for subject 4 in the "slope" grip (from $434 \mathrm{~N}$ to $236.38 \mathrm{~N}$ ). After twenty contractions, the mean force amounted to $270.49 \mathrm{~N}$ (SD: $39.33 \mathrm{~N}$ ) in the "crimp" grip and 279.25 N (SD: 61.34 N) in the "slope" grip. No significant difference was noted between these values $(p>0.05)$.
The force decrease amounted to $31 \%$ of the MVC value in the "crimp" grip, whereas it equalled $36 \%$ in the "slope" grip.

Negative $f_{\text {med }}$ slopes were observed in each subject except subject 1 who presents a slight $f_{\text {med }}$ slope increase (0.1) in the "slope" grip. A more pronounced $f_{\text {med }}$ slope was observed in subject 6 during the "crimp" grip (-1.41), whereas the statistical comparison of the mean $f_{\text {med }}$ does not indicate significant difference ( $p>0.05$ ). Fig. 2 illustrates the drop of $f_{\text {med }}$ and the slopes of the line of best fit are indicated for each grip. Negative mean $f_{\text {med }}$ slopes were exhibited in each grip, -0.76 (SD: 0.38) in the "crimp" grip and - 0.82 (SD: 0.62) in the "slope" grip, respectively.

\section{Discussion}

Most published handgrip forces in sport climbers range from $500 \mathrm{~N}$ to $700 \mathrm{~N}$ [5], with the exception of the study of Grant et al. [6]. The latter displayed fingertip forces around $450 \mathrm{~N}$, which is almost similar to the present data. Our results corroborate

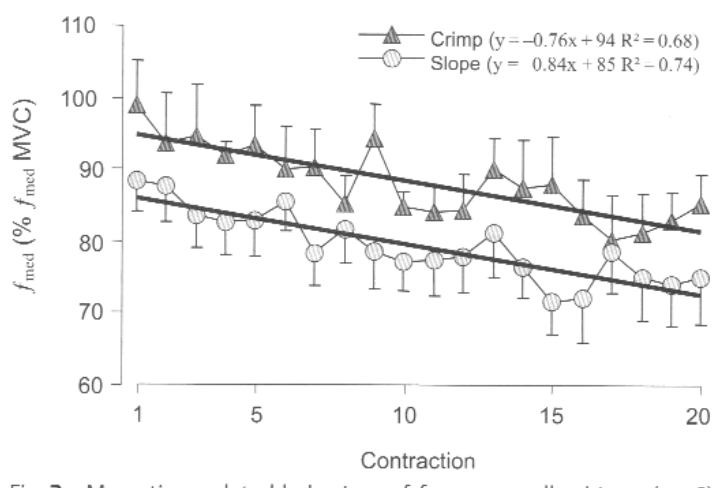

Fig. 2 Mean time-related behaviour of $f_{\text {med }}$ across all subjects $(n=6)$ for the "crimp" grip and the "slope" grip. Regression linear lines are superimposed for each grip. Vertical lines depict standard errors of the mean. 
Schweizer's [16] finding since the resultant four fingertip force does not change according to the sport climbing finger grip despite the subsequent biomechanical modifications. The FDS is responsible for flexion of the PIP joint, and the FDP is responsible for flexion of both DIP and PIP joints. In the "slope" grip, the moment arm of the flexor tendons increases with flexion of the DIP and PIP joints, increasing the muscular moment of the FDP and FDS muscles and consequently the holding force [16]. In a posture similar to the "crimp" grip, Dennerlein et al. [4] show that the force of FDP vanishes with the hyperextension of the DIP joint; therefore, they suggest that the FDS alone supports the holding force. The compensation of the inactive FDP seems to lie in the rise in the moment arm of the FDS over the PIP joint and/or probably in the increase in the FDS muscle tension. Nevertheless, although the inactivity of FDP was reported by Dennerlein et al. [4] using in vivo force measurement during low fingertip force intensity $(<10 \mathrm{~N})$, it is unclear whether this will hold true for greater fingertip force magnitude (i.e. $>10 \mathrm{~N}$ ) which can easily happen under loads at a fraction of bodyweight as in sport climbing. Moreover, several studies observed a common rupture of the finger A2 pulley system associated with the use of the "crimp" grip, whereas the "slope" grip does not present the same risks [15]. This is due to the different tendon moment arms associated with each sport climbing finger grip [16] and not to lower maximal resultant four fingertip forces applied in the "slope" grip. The practical consequence is that the use of the "crimp" or the "slope" grip during climbing does not seem to depend on the intensity of the resultant four fingertip force, but rather on other characteristics of grasping like size or painful injury risks associated with the hold.

The results of the maximal four fingertip force measured in the present study correspond to a laboratory experiment and may not necessarily be the same as during actual sport climbing. Some indications of the actual force placed on the fingers may be derived from previous studies conducted by Noé et al. [10] They employed a vertical/overhanging climbing frame instrumented with 3D force sensors to record force at each hand and foot support. Force at the fingers (one hand) amounted to $144 \mathrm{~N}$ during a quadrupedal state and $288 \mathrm{~N}$ after a foot hold release. These forces were considerably lower than the maximum finger forces recorded in the present experiment.

The results concerning muscular fatigue in the extrinsic flexors of the hand agree with several previous works [3,11]. They indicate that repetitive intense contractions induce a decrease in the fingertip force. Additionally, they show that the sport climbing finger grip does not affect the rate of force decrease. The similarity of the $f_{\text {med }}$ slopes and the 20 th force value in both sport climbing finger grips shows that the fatigue rate of flexor muscles is the same whatever the sport climbing finger grip.

Blackwell et al. [3] showed that fatigue rates are similar as long as the relative amounts of force are constant, regardless of the finger position. Contrary to the present data, Blackwell et al. [3] noted that the absolute maximal grip force differs according to the finger position (i.e. middle grip sizes produced greater absolute forces than the smallest or largest sizes) with less fatigue for middle grip sizes. This is not the case in the present study since similar absolute grip forces are observed in both grips. Conse- quently, the use of the "crimp" or the "slope" grip does not suggest any benefit with respect to muscular fatigue during sport climbing.

We conclude that the sport climbing finger grip does not affect the maximal resultant four fingertip force nor the fatigue rates of the extrinsic muscles of the hand. Hence, the finger grip force and the fatigue rates were not relevant in the decision made by climbers to use a "crimp" or a "slope" grip. Nevertheless, further examination of fingertip force and muscular fatigue is necessary in order to characterize the effect of alternation of the type of grip (crimp/slope) as is the case in sport climbing.

\section{References}

1 Basmajian JV, De Luca CJ (ed). Muscles Alive. Baltimore: Williams \& Wilkins, 1985: 94

2 Bigland-Ritchie B, Furbush F, Woods JJ. Fatigue of intermittent submaximal voluntary contractions: central and peripheral factors. J Appl Physiol 1986; 61: 421-429

${ }^{3}$ Blackwell JR, Kornatz KW, Heath ME. Effect of grip span on maximal grip force and fatigue of flexor digitorum superficialis. Appl Ergon 1999: 30: 401 - 405

${ }^{4}$ Dennerlein JT, Diao E, Mote CD, Rempel DM. Tensions of the flexor digitorum superficialis are higher than a current model predicts. J Biomech 1998; 31: 295-301

${ }^{5}$ Ferguson RA, Brown MD. Arterial blood pressure and forearm vascular conductance responses to sustained and rhythmic isometric exercise and arterial occlusion in trained rock climbers and untrained sedentary subjects. Eur J Appl Physiol 1997; 76: 174-180

${ }^{6}$ Grant S, Hynes V, Whittaker A, Aitchison T. Anthropometric strength, endurance, and flexibility characteristics of elite and recreational climbers. J Sports Sci 1996; 14: 301 - 309

${ }^{7}$ Hägg GM, Milerad E. Forearm extensor and flexor muscle exertion during simulated gripping work - an electromyographic study. Clin Biomech 1997: 12: 39-43

${ }^{8}$ Mermier MM, Robergs RA, McMinn SM, Heyward VH. Energy expenditure and physiological responses during indoor rock climbing. $\mathrm{Br} \mathrm{J}$ Sports Med 1997; 31: 224-228

${ }_{9}^{9}$ Mogk JP, Keir PJ. Crosstalk in surface electromyography of the proximal forearm during gripping task. J Electromyogr Kinesiol 2003; 13 : $63-71$

${ }^{10}$ Noé F, Quaine F, Martin L. Influence of steep gradient supporting walls in rock-climbing: biomechanical analyses. Gait Posture 2001; 13 : $86-94$

${ }^{11}$ Petrofsky JS. Quantification through the surface EMG of muscle fatigue and recovery during successive isometric contractions. Aviat Space Environ Med 1981: 52: 545 - 550

12 Pitcher JB, Miles TS. Influence of muscle blood flow on fatigue during intermittent human hand-grip exercise and recovery. Clin Exp Pharmacol Physiol 1997; 24: 471-476

${ }^{13}$ Quaine F, Martin L, Blanchi JP. The effect of body position and numbe of supports on wall reaction forces in rock climbing. J Appl Biomech 1997; 13: $14-23$

14 Quaine F. Vigouroux L, Martin L Finger flexors fatigue in trained rockclimbers and untrained sedentary subjects. Int J Sport Med 2003; 24:

$424-427$
15 Rooks MD. Rock climbing injuries. Sports Med 1997; 20: $261-270$

${ }^{16}$ Schweizer A. Biomechanical properties of the crimp grip position in rock climbers. J Biomech 2001; 34: 217-223

17 Stephens JA. Taylor A. Fatigue of maintained voluntary muscle con-

traction in man. J Physiol 1997; 220: 1 - 18
18 Watts PB, Dagget M, Gallagher P. Wilkins B. Metabolic response dur${ }^{18}$ Watts PB, Dagget M, Gallagher P, Wilkins B. Metabolic response dur-
ing sport rock climbing and the effects of active versus passive recovery. Int J Sports Med 2000; 21: 185-190 